\title{
Evaluation of a microdot immunofluorescent antigen detection test for Chlamydia trachomatis
}

\author{
C Thompson, R Jones, B Smith, O Brogan, I W Smith, D Carrington
}

\begin{abstract}
Objective-To evaluate a centrifuge enhanced direct immunofluorecent antigen test (MD test), compared with conventional culture and ELISA testing in the diagnosis of Chlamydia trachomatis infection.

Setting-A District General Hospital situated 30 miles from a University Department of Medical Microbiology. Subject and Method-A prospective study on specimens from 638 patients. Culture was performed on 348 specimens from genitourinary medicine patients and ELISA testing was carried out on 272 specimens from Gynaecology patients.

Results-When compared with culture the MD test had a sensitivity of $90 \cdot 6 \%$, specificity of $96.8 \%$, positive predictive value of $74.3 \%$ and a negative predictive value of $99 \%$. When compared with confirmed ELISA results the MD test had a sensitivity of $100 \%$, specificity of $98 \cdot 8 \%$, positive predictive value of $78.5 \%$ and negative predictive value of $100 \%$.

Conclusion-The MD test compares favourably with other chlamydial diagnostic techniques and in our setting is preferable to sending specimens for chlamydial culture. It is not suitable as the sole diagnostic method for screening large numbers of specimens but is a cost effective confirmatory test for positive ELISA results.
\end{abstract}

Department of

Genitourinary

Medicine, Victoria

Hospital, Kirkcaldy, Scotland

C Thompson

Fife Area Laboratory, Victoria Hospital, Kirkcaldy, Scotland

R Jones

B Smith

O Brogan

Clinical Virology

Laboratory,

University of

Edinburgh,

Department of

Medical Microbiology,

Edinburgh

I W Smith

Public Health

Laboratory, St

George's Hospital, London

D Carrington

Address correspondence to Dr C Thompson

Accepted for publication

7 April 1994
(Genitourin Med 1994;70:262-264)

\section{Introduction}

Chlamydia trachomatis is the commonest sexually transmitted bacterial infection seen in genitourinary medicine (GUM) departments in the UK. ${ }^{12}$ It is clinically important as the major cause of non-gonococcal urethritis (NGU) in men, cervicitis, salpingitis and infertility in women, and ophthalmia neonatorum and pneumonia in infants. ${ }^{3}$ There are several chlamydial diagnostic techniquesculture, direct immunofluorescent detection of antigen (IF) and the enzyme linked immunosorbent antigen assay (ELISA)none of which is infallible. ${ }^{4}$ In particular, chlamydial culture, although generally accepted as the "gold standard" has real disadvantages for specimens from outlying clinics and general practice surgeries because strict temperature conditions and rapid transport to the laboratory must be observed to ensure viability of the specimen. ${ }^{5}$ Direct methods of antigen detection are less labour intensive and it is for this reason that non-culture methods have become popular. However, the main drawback of these methods is that they are less specific than culture.

This study to evaluate a centrifuged enhanced direct immunofluorescent antigen test was carried out in a district general hospital laboratory (FAL) 30 miles $(48 \mathrm{~km})$ from the Clinical Virology Laboratory in the University of Edinburgh Department of Medical Microbiology (MME) where routine chlamydial culture and ELISA testing was performed. The transport service to $\mathrm{MME}$ was limited to three times a week and there were no facilities at FAL for storing material intended for chlamydial culture.

The object of our study was to choose a chlamydial diagnostic technique most suited to the needs of hospitals serving a semi-rural population of 340000 . To achieve this aim we prospectively compared the three accepted methods of diagnosis-culture, IF and ELISA, with an adaptation of the direct IF test (Syva MicroTrak, Palo Alto, Ca, USA) which has been applied to the detection of $C$ trachomatis antigen. This test was referred to as the microdot (MD) test for the immunofluorescent detection of $C$ trachomatis, and employed centrifugation to harvest chlamydial elementary bodies (EB) from the transport medium.

\section{Patients and methods}

For a four month period all clinicians (principally gynaecologists and genitourinary physicians) requesting a test for $C$ trachomatis were asked to take a second specimen where clinically possible, the order being alternated weekly. Specimens for processing by culture or $M D$ were taken from the urethra in men and the endocervix in women using cotton tipped plastic shaft swabs (Medical Wire and Equipment Co Ltd, Corsham, Wiltshire).

The swabs for culture were expressed into chlamydia transport medium (Northumbria Biologicals Ltd, Cramlington, UK) and stored at $4^{\circ} \mathrm{C}$ pending transport at ambient temperature to MME. The specimens were processed by single cycle culture on cyclohexamide treated McCoy cells. After 72 hours incubation chlamydial inclusions were identified by staining with iodine. Alternatively, specimens for ELISA testing (Chlamydiazyme, Abbott Diagnostics, Abbott Park, Illinois, USA) were taken using Abbott 
STD-EZE endocervical swabs and processed according to manufacturer's instructions. ${ }^{6}$ Positive ELISA results were confirmed by either IF (Syva MicroTrak), or the Abbott blocking antibody method. ${ }^{7}$ The IF test was also used for the examination of culture specimens contaminated with yeasts and was performed after microcentrifugation of the specimens. ${ }^{8}$ The other swab of the pair was broken off into the chlamydia transport medium and stored at $4^{\circ} \mathrm{C}$ at FAL until a batch of sufficient numbers was obtained (usually within 24 hours) for testing by the MD method.

\section{MD test method}

Samples were vigorously vortexed to release material from the swab and $0.5 \mathrm{ml}$ of the vortexed sample was transferred to a micro-centrifuge tube containing $0.5 \mathrm{ml}$ of distilled water and centrifuged for 10 minutes at $13,000 \mathrm{rpm}$ in a MSE Micro Centaur centrifuge. The supernatant was discarded and the remaining "pellet" re-suspended in $1 \mathrm{ml}$ of distilled water. The tubes were then vortexed to break up the "pellet" and centrifuged for a further 10 minutes at 13,000 rpm. Again the supernatant was removed leaving 50-100 $\mu \mathrm{l}$, enough to give a slightly milky suspension after vortexing. The larger the deposit the greater the suspension fluid required to avoid the subsequent transfer of excessive material for examination. Five microlitres of the suspension were placed onto a spot on a 15 spot slide (Multispot microscope slides, Hendley Essex, Loughton, Essex) and allowed to air dry at room temperature prior to fixing in methanol for 10 minutes. To avoid the possibility of cross contamination of consecutive wells, only alternate wells were used, thereby employing only 8 of the 15 wells, that is, 8 specimens per slide. Eight microlitres of directly conjugated monoclonal antibody (Syva MicroTrak) were placed on each test spot and the slide incubated in a moist chamber for 15 minutes at room temperature. The slides were then rinsed in distilled water, excess water dried from the slide by tilting the edge of the slide on blotting paper without touching the test area and mounted using glycerol/PBS anti-fade mountant (Syva MicroTrak). A coverslip was applied to the slide and the entire spot scanned using an oil immersion objective $(\times 63)$ on a Leitz incident light fluorescent microscope for the presence of fluorescent elementary and reticulate bodies of Chlamydia trachomatis. Positive and negative controls (Syva Microtrak) were included in each batch of tests. A positive test was recorded when 10 or more elementary

Comparison of microdot (MD) test performed at Fife area laboratory (FAL) with culture, ELISA and IF performed at University of Edinburgh Department of Medical

Microbiology (MME)

\begin{tabular}{|c|c|c|c|c|c|c|}
\hline \multirow[b]{2}{*}{$\begin{array}{l}\text { MD Result } \\
(N=633)\end{array}$} & \multicolumn{6}{|c|}{$M M E$ result by method of test } \\
\hline & $\begin{array}{l}\text { Culture } \\
\text { Positive }\end{array}$ & $\begin{array}{l}=348) \\
\text { Negative }\end{array}$ & $\begin{array}{l}\text { ELISA } \\
\text { Positive }\end{array}$ & $\begin{array}{l}=272) \\
\text { Negative }\end{array}$ & $\begin{array}{l}\text { IF }(n=13) \\
\text { Positive }\end{array}$ & Negative \\
\hline $\begin{array}{l}\text { Positive } \\
\text { Negative }\end{array}$ & 29 & $\begin{array}{r}10 \\
306\end{array}$ & $\begin{array}{r}11 \\
0\end{array}$ & $\begin{array}{r}3 \\
258\end{array}$ & $\begin{array}{l}1 \\
1\end{array}$ & $\begin{array}{l}3 \\
8\end{array}$ \\
\hline
\end{tabular}

bodies were found per specimen. If less than 10 were found in the first test another was prepared and re-tested to see if a cumulative count of ten or more elementary bodies could be obtained. If a cumulative count was still less than ten a result "EBs in low numberinconclusive" was recorded.

\section{Results}

Specimens from 638 patients were tested by both FAL and MME. Five of the FAL results detected low number of EBs (reported by MME as positive in all five cases) and these results have been excluded from further analysis. The diagnostic method used at MME and the results from both FAL and MME for the remaining 633 patients are shown in the table.

Overall $41(6 \cdot 4 \%)$ specimens were reported positive by both laboratories. There were 20 (3\%) discrepant results: 16 positive $M D$ tests were reported negative by MME of which 10 were processed by culture, three by ELISA and three by IF; four negative MD tests were positive at MME, three by culture and one by IF.

The MD test results were compared separately with the culture and confirmed Elisa results. A formal comparison with IF was not made as the numbers were so small.

\section{Discussion}

Reliable chlamydial diagnosis is inherently compromised by organism viability; the subjectivity of microbiological interpretation and the potential for false reactivity in solid phase tests. There is no universally accepted "gold standard", although chlamydial culture is the usual method against which other tests are compared. The direct MicroTrak test examining direct smears on slides is, in some centres, more reliable than ELISA, ${ }^{4}$ but is practical for only small numbers of samples. For logistic reasons, clinicians in Fife had used two different tests, thus enabling the new MD technique to be compared with both culture and ELISA. Owing to problems with storage and transport to a laboratory 30 miles away, culture was generally used only for genitourinary medicine (GUM) patients, as they are a higher prevalence group. A negative result in this context was of doubtful validity in view of the distance from the laboratory (MME). The ELISA test was used for non-GUM (mainly gynaecology) patients for two reasons (a) there was the problem of storage and transport of culture specimens and (b) the turnaround time required was shorter than that which could be achieved by culture. A confirmatory technique (either blocking antibody or IF) was used to over come the problem of false-positive results.

Comparison with culture of the MD test gives a sensitivity of $90.6 \%$; specificity of $96.8 \%$; positive predictive value of $74.3 \%$ and negative predictive value of $99 \%$ for the MD test. In reality the sensitivity may be higher than this: the case notes of those patients reported 
as $\mathrm{MD}$ positive/culture negative were examined and three were found to have $C$ trachomatis culture positive contacts; thus three of the discrepant results may represent false negative culture results rather than false positive $\mathrm{MD}$ results. This may possibly be due to problems inherent in transporting viable specimens for culture from a peripheral unit.

Comparing with confirmed ELISA results, the MD test has a sensitivity of $100 \%$ and a specificity of $98.8 \%$, positive predictive value of $78.5 \%$ and negative predictive value of $100 \%$. The MD test with an overall sensitivity greater than $90 \%$ and specificity greater than $95 \%$, compares favourably with other chlamydial diagnostic techniques. It has the advantage that specimens are readily stored for "batch" testing which is both time saving and a more cost effective routine. It is particularly suitable for clinicians working in outlying areas. The use of a microfuge gives $g$ forces very close to high speed centrifugation, but in a fraction of the time, and is therefore faster and more convenient than a bench centrifuge. By concentrating and harvesting organisms from the clinical specimen, the $M D$ test has enhanced sensitivity compared with the direct MicroTrak. ${ }^{8}$ The microdot method also provides the following advantages over direct examination of smears: $(a)$ a washing procedure to remove mucoid material, $(b)$ more cost efficient use of monoclonal antibody, $(c)$ smaller area of examination of deposit than usual with a smear, $(d)$ Elementary bodies all in same plane of visualisation during microscopy, (e) re-examination of deposit for multiple sampling is possible.

The multiwell slide used for the MD test makes handling easier for the MLSO, and cross contamination from well to well has not been shown to be a problem. If contamination is suspected, for example when a low positive is situated close to a high positive on the original slide, a repeat test from the deposit can be performed. Because the method involves direct microscopy the amount of cellular material can be assessed and this may be of benefit in evaluating the quality of specimens provided. These advantages should be particularly helpful to smaller laboratories processing a limited number of samples or to larger laboratories where urgent samples, such as ophthalmic swabs, nasopharyngeal aspirates, require individual processing outside ELISA batch routines. The disadvantage of the $\mathrm{MD}$ method is that, in large numbers, it is laborious to perform and depends on highly experienced staff being able to recognise the characteristic fluorescence pattern of elementary bodies. It is therefore less suitable than ELISA for the sole testing of large numbers of specimens.

However, after initial screening by an ELISA method, the MD method is a costeffective confirmatory test for positive ELISA results. In comparative tests using several commercially available ELISA kits, the microdot test shows equal sensitivity (personal observations), including kits utilising enzyme amplification.

We conclude that in our setting, the $\mathrm{MD}$ test, with a negative predictive value compared with culture of $99 \%$, and a positive predictive value compared with ELISA of $78 \%$ was preferable to sending specimens for chlamydial culture. The ability of FAL to process a larger number of samples might be improved by the introduction of an ELISA method for initial screening of all specimens with subsequent confirmation of positive ELISA results by the MD method.

In view of the recognised inaccuracies inherent in non-culture techniques it is, however, recommended that in any case with potential legal involvement, for example rape, child sexual abuse, specimens for culture be taken in addition to direct antigen tests.

1 PHIS CDC Sexually transmitted disease in Britain 1985-6. Genitourin Med 1989;65:117-21.

2 Information and Statistics Division, Scottish Health Service, Common Services Agency, Edinburgh. Sexually transmitted diseases Statistics 1990

3 Robertson DHH, McMillan A, Young H. Non-gonococca urethritis, chlamydial infections and other related conditions. In: McMillan A, Young H, eds. Clinical Practice in Sexually Transmissable Diseases, 2nd ed. Edinburgh: Churchill Livingstone, 1989.

4 Ridgway GL, Taylor-Robinson D. Current problems in microbiology: 1. Chlamydial infections: Which labora-

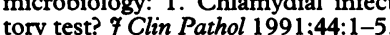

5 Taylor-Robinson D, Thomas BJ. Laboratory techniques for the diagnosis of chlamydial infections. Genitourin for the diagnosis of

6 McKenna JG, Young H, Moyes A, Smith IW. Is co-existing chlamydial infection more common in gonococcal infections with serogroup WI? Int $\mathcal{f}$ STD AIDS 1990;1: $340-2$.

7 Moncada J, Schachter J, Bolan G, et al. Confirmatory Assay Increases Specificity of the Chlamydiazyme tes for Chlamydia trachomatis infection of the cervix. $\mathcal{F}$ Clin Microbiol 1990;28:1770-3.

8 Thomas B, Gilchrist C, Taylor-Robinson D. Detection of Chlamydia trachomatis by Direct Immunofluorescence Improved by Centrifugation of Specimens. Eur $¥$ Clin Microbiol Infect Dis 1991;10:659-62 